\section{Medicina hospitalaria a 15 años de su implementación. ¿Cuáles son los potenciales beneficios en nuestro medio?}

\author{
GONZALO EYMIN ${ }^{1,2}$, AMIR K JAFFER ${ }^{2}$
}

\section{Hospital medicine in Chile}

\begin{abstract}
After 15 years of development of Hospital Medicine in Chile, there are several benefits of this discipline. Among others, a reduction in the length of hospital stay, readmissions, costs, and improved medical teaching of students, residents and fellows have been observed. However, in South America there are only isolated groups dedicated to Hospital Medicine in Chile, Argentina and Brazil, with a rather slow growth. The unjustified fear of competition from sub specialists, and the fee for service system of payment in our environment may be important factors to understand this phenomenon. The aging of the population makes imperative to improve the safety of our patients and to optimize processes and resources within the hospital, to avoid squandering healthcare resources. The following is a detailed and evidence-based article, on how hospital medicine might benefit both the public and private healthcare systems in Chile.
\end{abstract}

(Rev Med Chile 2013; 141: 353-360).

Key words: Home care services, hospital-based; Hospitalists; Hospital medicine.

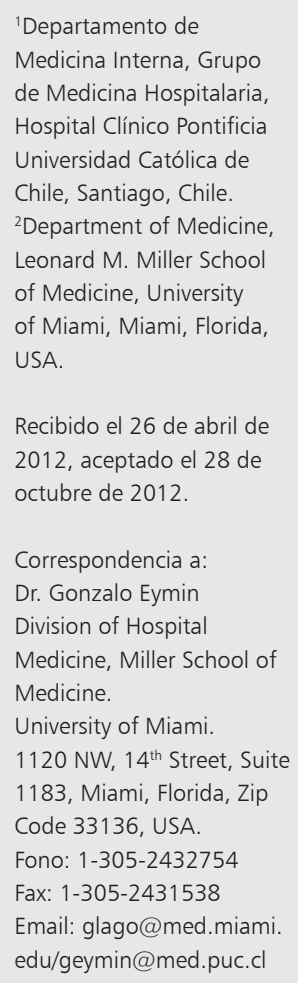

Recibido el 26 de abril de 2012, aceptado el 28 de octubre de 2012.

Correspondencia a:

Dr. Gonzalo Eymin

Division of Hospital

Medicine, Miller School of

Medicine.

University of Miami.

$1120 \mathrm{NW}, 14^{\text {th }}$ Street, Suite

1183, Miami, Florida, Zip

Code 33136, USA.

Fono: 1-305-2432754

Fax: 1-305-2431538

Email: glago@med.miami.

edu/geymin@med.puc.cl

L a medicina hospitalaria es una disciplina dedicada a entregar cuidados médicos exhaustivos a pacientes hospitalizados, a la vez que se encarga de la docencia clínica, investigación y liderazgo en el campo de la medicina interna. A diferencia del internista general, el hospitalista brinda una atención completa y oportuna de todas las necesidades del paciente, incluyendo diagnóstico, tratamiento y procedimientos de variada índole, incorpora técnicas de mejoramiento de procesos y de calidad, colabora, comunica y coordina los cuidados prestados por los diferentes profesionales de la salud, hace uso eficiente de los recursos hospitalarios y se encarga de que las transiciones del paciente a través del hospital y entre este y su domicilio sea segura ${ }^{1}$. Sus competencias son variadas, y se pueden agrupar en condiciones clínicas, procedimientos y en los sistemas al interior del hospital (seguridad de pacientes, docencia, mejoría de procesos). Esta especialidad, descrita en Estados Unidos de Norteamérica en 1996, ha alcanzado un desarrollo y crecimiento sin precedentes en ese país, superando el crecimiento de todas las especialidades de la medicina ${ }^{2-4}$, crecimiento que coincide con el cambio en la forma de reembolso de las prestaciones médicas ${ }^{5}$, y con el creciente aumento del interés por mejorar la seguridad en los hospitales después de la publicación "To err is human" (1999) en la cual se describe que entre 44.000 y 98.000 personas mueren cada año en Estados Unidos de Norteamérica producto de eventos adversos potencialmente prevenibles ${ }^{6}$. Antiguamente el pago de prestaciones de salud en Estados Unidos de Norteamérica se hacía por servicios prestados (Fee for service), sin embargo, actualmente se hace en relación a un determinado grupo de diagnósticos relacionados, en el cual todas las condiciones codificadas bajo un mismo grupo son reembolsadas con un monto fijo independientemente del curso hospitalario del paciente ${ }^{7}$. Así, si los hospitales acortan las estadías y realizan menos exámenes, el ahorro para el hospital es mayor. Además, a partir del 2010 el CMS (Entidad pagadora de prestaciones en salud) comenzó a negar el reembolso asociado a reingresos a 30 días en pacientes con neumonía, insuficiencia cardiaca, 
infarto del miocardio, y tromboembolismo después de artroplastias de cadera y rodilla ${ }^{8,9}$. Todo esto, sumado a la aparición de sitios como http:// hospitalcompare.com en el cual se comparan los resultados de distintos hospitales, propició las condiciones para el surgimiento de la medicina hospitalaria, disciplina que rápidamente permeó en los hospitales dada su eficiencia en disminuir la duración de las estadías hospitalarias, los costos, los reingresos y los errores mediante la estandarización de los procesos ${ }^{10,11}$. No obstante, esta disciplina ha permeado lentamente en Chile, con sólo unos pocos grupos de médicos en algunos hospitales. El gasto en salud per cápita en Chile expresado en dólares, es 7 veces menor que en Estados Unidos de Norteamérica $^{12}$, sin embargo, el lento progreso en nuestros países va más allá del aspecto económico. En nuestra cultura desde siempre los médicos han hecho las cosas de la manera que su experiencia les dicta, y no de forma estandarizada y apoyada en evidencia. Personal paramédico, por otro lado, (muchas veces funcionarios con decenas de años de servicio), son reacios a cambiar la forma en que se hacen las cosas, y cada intervención es tomada como un bache más en el trabajo, más que como una oportunidad para mejorar ${ }^{13}$. A esto hay que sumar el temor de sub-especialistas que ven a los hospitalistas como competencia.

Cuando se evalúan ciertos estándares básicos de muchos hospitales, podemos percatarnos que estamos lejos de tener una cultura de seguridad y de optimización de procesos. El crecimiento de nuestra salud no pasa por construir más hospitales, sino que por una reformulación y reingeniería de los procesos hospitalarios, tal como sucedió con la medicina ambulatoria en los últimos años ${ }^{14}$. Los costos asociados a la implementación de la medicina hospitalaria es otro factor a considerar en el lento desarrollo de esta disciplina en nuestro país, ya que los ingresos del hospitalista se generan casi exclusivamente de la actividad hospitalaria. Sin embargo, los hospitales pueden compensar esta inversión con una disminución en los tiempos de estadía y en la tasa de reingresos. Hay que considerar que más allá de los costos, el hospitalista agrega valor al mejorar la calidad de la atención sin aumentarlos. Otro factor que puede estar asociado a este lento crecimiento es la falta de reconocimiento de las competencias que un médico internista "general" pueda tener para manejar una gran gama de pacientes hospitalizados.
La introducción de médicos especialistas en estandarización de procesos, con conceptos de seguridad arraigados, exige un cambio en la estructura organizacional y de convivencia en los servicios hospitalarios. Todos los cambios implementados deben pasar a formar parte del flujo habitual de trabajo de modo de evitar que la gente pronto comience a trabajar esquivando la norma, y debe darse a conocer al personal de salud las razones que hacen necesario el cambio impuesto de modo de ir consolidando una cultura de seguridad y de calidad. La labor del hospitalista es por definición multidisciplinaria, y entender que todas las personas tienen derecho a opinar es el pilar fundamental a la hora de formar un buen equipo. El hospitalista no puede optimizar la prestación de servicios sin contar con un equipo de trabajo comprometido que vele por los mismos intereses, es decir, el paciente por un lado y el hospital por el otro. De hecho, el programa nacional de salud para los próximos 10 años habla de fortalecer los equipos de trabajo y mejorar sus condiciones laborales ${ }^{15}$.

\section{Mejoría de los procesos}

El hospitalista, debiéndose al paciente por un lado y al hospital por el otro, debe velar por la seguridad del paciente y por la optimización de los procesos. La presencia de hospitalistas es una gran ventaja para los centros que desarrollan proyectos de "quality improvement" (mejoría de calidad), puesto que la cobertura y el contacto con personal ancilar casi permanente, asociado a las habilidades de liderazgo, lo dejan en una posición única para solucionar en tiempo real problemas que puedan aparecer. White mostró que en $71 \%$ de 65 artículos revisados, los hospitalistas mejoraron la calidad ${ }^{16}$. Rifkin encontró que pacientes con neumonía manejados por hospitalistas eran más susceptibles de recibir prevención de enfermedad tromboembólica y vacuna antineumocócica $(\mathrm{p}<0,001)^{17}$. Lindenauer reportó que los hospitalistas documentaban la fracción de eyección en pacientes con insuficiencia cardiaca con mayor frecuencia que no hospitalistas $(\mathrm{p}=0,04)^{18}$. Roytman demostró una reducción de la estadía hospitalaria, un mayor uso de inhibidores de la ECA o antagonistas del receptor de angiotensina, un mayor uso de diuréticos IV, y menores costos en pacientes con insuficiencia cardiaca manejados por hospitalistas 
$(p<0,003)^{19}$. Sin embargo, el hospitalista no ha demostrado beneficios en pacientes con VIH ni en pacientes con dolor torácico ${ }^{20,21}$. No hay estudios que comparen la demora en la entrega de atención médica, la calidad de las entregas de turno y las altas entre hospitalistas y no hospitalistas. No hay estudios que comparen el impacto del hospitalista en la mejoría de procesos versus servicios manejados con internistas generales de la mano de expertos en gestión clínica.

\section{Duración de estadías hospitalarias y reingresos}

La estadía hospitalaria promedio en Chile es de 5,8 días, cifra similar a la de otros países. Sin embargo, no contamos con estadísticas claras respecto a reingresos. Dado el gran volumen de pacientes que absorbe el sistema de salud, incluso pequeñas reducciones en la duración de las estadías tendría un tremendo impacto económico a nivel global ${ }^{7}$. A modo de ejemplo, un estudio en un hospital público nacional mostró que $41 \%$ de los pacientes tuvo estadías hospitalarias más largas de lo esperado ${ }^{22}$, y puesto que el hospitalista conoce los factores de riesgo para hospitalizaciones prolongadas y reingresos, su gestión puede conllevar a hacer un uso más adecuado de los recursos de salud ${ }^{23,24}$. El hospitalista es el médico en mejor posición para acortar las hospitalizaciones, puesto que al tener presencia permanente en el hospital puede dar de alta pacientes a casi cualquier hora, evitando postergarla hasta el día siguiente en espera de algo pendiente, cosa que médicos con menor presencia en el hospital, incluso teniendo formación en gestión clínica probablemente no son capaces de hacer en forma sistemática ${ }^{7}$. White encontró una disminución significativa en la duración de la estadías en 60\% de los 48 estudios evaluados, cifra que se eleva a $88 \%$ cuando se compara a hospitalistas académicos con médicos de la comunidad ${ }^{16}$. Dos estudios encontraron una disminución en la duración de la estadía hospitalaria en pacientes con neumonía manejados por hospitalistas versus no hospitalistas $(\mathrm{p}=0,001 \text { y } \mathrm{p}<0,004)^{25,26}$. Si bien los estudios de Meltzer y de Auerbach en servicios de medicina no demostraron una disminución del tiempo de estadía en el primer año de implementación de la medicina hospitalaria, si lo lograron en el segundo año $(\mathrm{p}=0,001 \text { y } \mathrm{p}=0,002)^{27,28}$, hecho que podría explicarse por la fuerte correlación que existe entre experiencia y la calidad de la atención entregada, tal como ha sucedido en las unidades de cuidados intensivos, y en otras áreas ${ }^{29}$. Sin embargo, con el fin de no tener un rebote en la tasa de reingresos, es crucial considerar como es la comunicación con los proveedores primarios de salud antes de implementar iniciativas destinadas a reducir la duración de las hospitalizaciones ${ }^{30}$.

\section{Infecciones asociadas a la atención en salud (IAAS)}

Las IAAS, la mayoría de las cuales son prevenibles, provocan 90.000 muertes al año sólo en Estados Unidos de Norteamérica ${ }^{31}$, y las infecciones del torrente sanguíneo adquiridas en el hospital son la octava causa de muerte en ese país ${ }^{28}$. Las medidas necesarias para evitar las IAAS en relación a catéteres venosos centrales, catéteres urinarios y ventilación mecánica son ampliamente conocidas $y$ difundidas ${ }^{32-34} y$, en teoría, la presencia del hospitalista favorecería la diseminación, adherencia y consolidación de éstas para disminuir la tasa de IAAS, puesto que su mayor presencia y nivel de alerta respecto a esto le permitiría trabajar en forma sistemática y continua con todos los miembros del equipo de salud.

\section{Docencia}

El hospitalista es el médico que más tiempo pasa con estudiantes, internos y residentes en el entrenamiento de destrezas en el manejo de pacientes hospitalizados. En la medida que los hospitalistas reemplazan a sub-especialistas en el manejo de pacientes hospitalizados, se refuerza el concepto de manejo clínico general integral. Varios son los estudios que demuestran la preferencia de residentes por los hospitalistas en cuanto a satisfacción, calidad de las visitas, experiencia educativa, conocimientos del manejo intrahospitalario, discusiones de fisiopatología, énfasis en costo efectividad y feed-back $(\mathrm{p}<0,05)^{35-37}$. Los estudiantes de medicina consideran que los hospitalistas proveen de una mejor experiencia educativa, de visitas más docentes y entusiastas, usan más la evidencia, modelan mejor los buenos modales, y tienen mayor presencia y compromiso 
con los pacientes $(\mathrm{p} \leq 0,03)^{38,39}$. El meta-análisis de Natarajan que incluye estudios en estudiantes y residentes tiene conclusiones similares ${ }^{40}$.

\section{Manejo peri-operatorio}

En el meta-análisis de White se encontró una disminución en el tiempo de espera a cirugía de 35 a 50\% cuando la admisión y evaluación preoperatoria era realizada por hospitalistas versus cirujanos, sin reducción en los $\operatorname{costos}^{16}$. Huddleston demostró una reducción en las complicaciones post operatorias en pacientes ortopédicos manejados por hospitalistas $(\mathrm{p}<0,01)^{41}$. Phy y Roy encontraron que pacientes ortopédicos co-manejados por hospitalistas tuvieron una latencia menor a cirugía ( $\mathrm{p}<0,001$ y $\mathrm{p}=0,004)^{42,43}$, y estadías hospitalarias más cortas que los pacientes manejados sin hospitalistas $(\mathrm{p}<0,001)^{42}$.

\section{Satisfacción}

Wachter comparó un sistema tradicional de un servicio de medicina con uno hospitalista, encontrando una tendencia a una mayor satisfacción tanto en médicos de planta como en residentes $(\mathrm{p}=0,06)$, reconociendo ambos que el modelo hospitalista hacía mucho más énfasis en conceptos de costo-efectividad $(\mathrm{p}<0,001)^{44}$. Wachter describe que la satisfacción de pacientes no se deteriora con el modelo hospitalista ${ }^{7}$.

\section{Mortalidad}

En 20\% de los estudios en el meta-análisis de White, se encontró una disminución de la mortalidad en pacientes manejados por hospitalistas ${ }^{16}$, hallazgo también descrito por Peterson ${ }^{45}$. Si bien la calidad metodológica de los estudios no es la mejor, el estudio randomizado de Meltzer encontró una menor mortalidad a 30 días en pacientes manejados por hospitalistas en el segundo año de implementación del programa $(\mathrm{p}=0,04)^{28}$.

\section{Costos}

En el meta-análisis de White 63\% de los estudios mostraron una reducción de los costos con los hospitalistas, beneficio también encontrado por Meltzer y Auerbach a partir del segundo año de implementación del programa hospitalista $(\mathrm{p}=0,01$ y $\mathrm{p}=0,002)$, lo cual sugiere fuertemente que estos resultados se van logrando en equipos con experiencia ${ }^{27,29,35}$. En pacientes con neumonía, Rifkin y Scheurer encontraron una disminución de los costos en pacientes manejados por hospitalistas $(\mathrm{p}=0,01 \mathrm{y} \mathrm{p} \leq 0,003)^{25,26}$. Gregory sugiere que los beneficios económicos de la medicina hospitalaria se deben a que se mejora el flujo de pacientes, optimizándose el rendimiento ${ }^{46}$. Por otro lado, Diamond encontró que casi todo el ahorro se hace en las patologías de mayor nivel de consumo, lo cual es coherente con que la mayor reducción en la duración de las estadías se logró en aquellas patologías con mayor estancia promedio . $^{8}$

\section{Uso de sub-especialistas}

Peterson encontró en su meta-análisis una disminución en el requerimiento de interconsultas en pacientes manejados por hospitalistas ${ }^{45}$. En la medida que el manejo de pacientes en el hospital se profesionaliza, el requerimiento de interconsultas podría disminuir, lo cual ofrece una atractiva solución en lugares del país en que aun hay escasez de sub-especialistas.

\section{Desafíos de la medicina hospitalaria}

La llegada del hospitalista introduce discontinuidad a los cuidados, aumentando teóricamente el riesgo de errores médicos y eventos adversos durante las transiciones ${ }^{47}$. Esto es particularmente importante en nuestro sistema público de salud, en el que existe un abismo entre la atención primaria y los hospitales, por lo tanto, mejorar la comunicación y el flujo entre hospitalistas y médicos primarios es crucial. Por otro lado, el hospitalista no siempre está al tanto de las preferencias del paciente y su familia en relación a la resucitación y otras medidas de rescate, de ahí que sea importante intercambiar información con el médico primario al ingreso, al alta y cuando la condición clínica del paciente lo amerite ${ }^{48}$. Para el Dr. Wachter, la presencia del médico primario en la discusión sobre medidas de resucitación con la familia es invaluable, y concluye que el hospitalista y el médico primario deben actuar 


\section{Tabla 1. Objetivos del gobierno de Chile para el período 2011-2020 y como el hospitalismo podría ayudar en su cumplimiento}

\section{Plan y comentarios Minsal ${ }^{12}$}

Se consolida la información estadística oficial el sector salud, y se cuenta con registros continuos de mortalidad, morbilidad, recursos, atenciones de la red asistencial y de la autoridad sanitaria entre otros

La población mayor de 60 años se proyecta en $14,8 \%$ para 2015 y 17,3\% para 2020. Se estima que un poco más de la mitad del gasto en salud en adultos mayores corresponde a gastos en hospitalización

La utilización de Grupos Relacionados de Diagnósticos Refinados Internacionales (IR-GRD) y un sistema de asignación de recursos por costos directos e indirectos, se inserta en la formulación de un Programa Clínico Financiero. Para los próximos años se pretende conocer el GRD involucrado en el 80\% de los egresos hospitalarios

Aumentar los establecimientos de salud acreditados

El mejorar las dimensiones de calidad tendrá efecto en grandes metas sanitarias, como la reducción de la mortalidad, morbilidad, y el aumento de la satisfacción del usuario

La calidad y seguridad de la atención en hospitales es una preocupación central para los sistemas de salud. El único evento adverso sujeto a vigilancia permanente actual, son las Infecciones Asociadas a la Atención Sanitaria (IAAS)

Durante los últimos años, se han realizado diversas estrategias en pos de la mejoría de la calidad y seguridad de la atención de los pacientes en el ámbito hospitalario. Se ha avanzado en modificaciones legales para la estandarización de los procesos para el sector público y privado, mediante la ley 19.337 de Autoridad Sanitaria del 2004, y la modificación de los reglamentos de Hospitales y Clínicas y de Salas de Procedimientos y Pabellones de Cirugía Menor del 2006. Las estrategias se han concentrado en la generación de protocolos de atención basados en evidencia, por ejemplo: normas de programas de salud, guías de práctica clínica y protocolos. Ejemplos de esto son Programa de Prevención y Control de Infecciones Intrahospitalarias, la implementación de indicadores de seguridad de la atención para cirugía (lista de chequeo quirúrgico, prevención de la enfermedad tromboembólica y análisis de re intervenciones no programadas), y para la atención y cuidados de pacientes (prevención de úlceras por presión y caídas de pacientes) y para medicina transfusional

En el ámbito del uso de los principios de la medicina basada en la evidencia en la práctica clínica, se propone la elaboración de orientaciones técnicas para la formulación de guías y protocolos GES y no GES

Se incorporó en los Compromisos de Gestión de los Servicios de Salud, la elaboración e implementación de Planes Integrales de Mejora del Trato que impacten tanto en la satisfacción usuaria como en los reclamos interpuestos por la población usuaria

\section{Relación con el hospitalismo}

Necesidad de mejorar los procesos y la seguridad de pacientes. Resultados de los hospitales podrán ser comparados

Junto con fortalecer la prevención, esta es una gran oportunidad para reducir los costos hospitalarios

Pago asociado a patologías. Uno de los puntapiés en el inicio del hospitalismo. Necesidad de mejorar los procesos y contener los costos ante ingresos fijos

Estandarización de los procesos y trabajo en equipo en terreno. Incentivar la cultura de seguridad y calidad

Cultura de seguridad y calidad y estandarización de procesos, con base en la evidencia

Los procesos de acreditación se focalizan en la calidad y seguridad asistencial. Como se describió en el manuscrito este es el centro de la labor hospitalista. La acreditación exige muchos otros puntos aparte de las IAAS

La estandarización de los procesos con base en la evidencia son los pilares en cada una de estas intervenciones. La presencia permanente del hospitalista en el hospital junto a su liderazgo, lo dejan en una posición privilegiada para velar que los protocolos se cumplan

La sola creación de guías clínicas y protocolos no garantiza su éxito. Un trabajo permanente en terreno es mandatorio para el éxito de lo anterior

El liderazgo del hospitalista ofrece una gran oportunidad para formar equipos de trabajo cohesionados que velen por la mejor atención posible. Su rol en crear una cultura de calidad y seguridad es el mejor aliado para alinear a los trabajadores en brindar una atención respetuosa 
como un equipo para cuidar de forma efectiva al paciente en su transición hacia su hogar o hacia el hospital ${ }^{49}$. Por otro lado, el burn-out es un factor a considerar. A juicio del Dr. Wachter, 12 meses como hospitalista en práctica clínica es la fórmula para el burn-out, siendo 3 a 6 meses al año más sostenible en el tiempo, tal como sucedió con los intensivistas, realidad difícil de implementar en nuestro medio ${ }^{29}$.

En la Tabla 1 se visualizan los objetivos del gobierno de Chile para el período 2011-2020 y como el hospitalismo podría ayudar en su cumplimiento.

\section{Conclusión}

El actual escenario en salud, con una población envejecida y con múltiples patologías crónicas hace primordial contar con hospitalistas. Esto, que es bastante evidente en el sector público de salud, no lo es tanto en el sector privado. Si bien el sector privado aparentemente no se beneficia en reducir la duración de las estadías hospitalarias o los costos, si lo haría mejorando la seguridad y optimizando los procesos. El disminuir los eventos adversos y reducir los costos sin alterar la satisfacción del paciente es la mejor propaganda que se puede tener. Se sabe que una opinión positiva puede acercar muchos usuarios, sin embargo, una negativa puede alejar aun a más. Si bien en nuestro medio no existen recursos como hospitalcompare. com, siguiendo la tendencia en Estados Unidos de Norteamérica, no es raro que esto suceda en el futuro, lo cual pone de relieve la necesidad de mejorar los procesos desde ya. En caso de proliferar el modelo, no estamos preparados para suplir la demanda, y en este sentido, en vez de contar con una nueva especialidad, se podría otorgar al tercer año de la residencia en medicina interna un enfoque más hospitalario, con especial énfasis en aspectos de calidad y seguridad asistencial. Hay que dejar en claro que el hospitalista no compite con el subespecialista. Si bien en lugares con escasez de estos pueden ser una solución, en grandes centros hospitalarios su rol es ser el director de orquesta durante la hospitalización de los pacientes, encargándose de ámbitos tan prevalentes como son la prevención de tromboembolismo, manejo del delirium, diabetes y dolor, prevención de IAAS y aplicación de guías clínicas en el manejo de patologías frecuentes. Además podrían ser de gran ayuda en el manejo de pacientes quirúrgicos y en la docencia de estudiantes de medicina. En este mismo sentido hay que dejar en claro que el hospitalista no es un experto en gestión clínica, sino que es un operador con arraigados conceptos de gestión en su quehacer diaria, y por lo tanto, no debe ser visto como la única solución a los problemas en la administración hospitalaria. Sin una inversión inicial, con un apoyo gubernamental e institucional robusto, es difícil que el hospitalismo pueda diseminarse. Si bien el plan de salud del gobierno para el período 2010-2020 no hace mención alguna al hospitalismo, si cuenta con varios puntos que atañen directamente a la administración hospitalaria, la seguridad de pacientes y optimización de los procesos, y de este modo dejan la puerta abierta para que se pueda implementar el modelo en el mediano plazo. En la medida que se les exija a los directores de hospitales cumplir con metas exigidas por agencias de salud como Joint Commission, el hospitalista va a tomar el real valor que merece en nuestro medio. Por último cada centro debería implementar medidas para evitar el burn-out de sus hospitalistas, de modo de hacer el sistema viable en el tiempo.

\section{Referencias}

1. SHM Board. Definition of a Hospitalist and Hospital Medicine. November 2009. Accesed August $12^{\text {th }}$ 2012. Available from: http://www.hospitalmedicine.org/AM/Template.cfm?Section=Hospitalist_ Definition \&Template $=/ C M /$ HTMLDisplay.cfm \& ContentID $=24835$.

2. Auerbach A, Chlouber R, Singler J, Lurie JD, Bostrom A, Wachter R. Trends in market demand for internal medicine 1999 to 2004: an analysis of physician job advertisements. J Gen Intern Med 2006; 21 (10): 1079-85.

3. Kralovec P, Miller J, Wellikson L, Huddleston J. The status of hospital medicine groups in the United States. J Hosp Med 2006; 1 (2): 75-80.

4. Hinami K, Whelan C, Wolosin R, Miller J, Wetterneck T. Worklife and satisfaction of hospitalists: toward flourishing careers. J Gen Intern Med 2012; 27 (1): 28-36.

5. Centers for Medicare and Medicaid Centers. Acute inpatient PPS. Accesed August $12^{\text {th }}$ 2012. Available from: http://www.cms.gov/Medicare/Medicare-Fee-for-Service-Payment/AcuteInpatientPPS/index.html?redirect=/ AcuteInpatientPPS/ 
6. Kohn L, Corrigan J, Donaldson M. Institute of Medicine. To Err is Human:Building a safer health system. Washington, USA: National Academy of Sciences, 2000.

7. Organization for Economic Co-operation and Development. OECD Health Data 2011 - Frequently Requested Data. 2011 February 17th 2012]; Available from: http://www.oecd.org/document/16/0,3746, en_2649_33929_2085200_1_1_1_1,00.html.

8. Coye M. CMS' stealth health reform. Plan to reduce readmissions and boost the continuum of care. Hosp Health Netw 2008; 82 (11): 24.

9. Streiff M, Haut E. The CMS ruling on venous thromboembolism after total knee or hip arthroplasty: weighing risks and benefits. JAMA 2009; 301 (10): 1063 5.

10. Wachter R, Goldman L. The hospitalist movement 5 years later. JAMA 2002; 287 (4): 487-94.

11. Diamond H, Goldberg E, Janosky J. The effect of fulltime faculty hospitalists on the efficiency of care at a community teaching hospital. Ann Intern Med 1998; 129 (3): 197-203.

12. Organization for Economic Co-operation and Development. Total expenditure in health per capita, at current prices ad PPPs. 2011 February 23rd, 2012]; Available from: http://www.oecd-ilibrary.org/socialissues-migration-health/total-expenditure-on-healthper-capita_20758480-table2.

13. Muñoz P. Percepción y resistencia al cambio del usuario interno de un establecimiento de atención primaria, en el contexto del proceso de transformación del modelo de salud familiar. 2007. Accedido 31 de marzo de 2012. Available from: http://medicina.uach.cl/saludpublica/ diplomado/contenido/trabajos/1/Osorno\%202007/ PERCEPCION\%20Y\%20RESISTENCIA\%20AL\%20 CAMBIO\%20DEL\%20USUARIO\%20INTERNO\%20 DE\%20UN\%20ESTABLECIMIENTO\%20DE\%20 ATENCION\%20PRIMARIA.pdf.

14. Bozzo G. Desarrollo de la Medicina Familiar en Chile. Accedido 31 de marzo de 2012. Available from: http:// www.medicinafamiliar.cl/sitio/index.php?option=com content\&view=article\&id=118\&Itemid=65.

15. Gobierno de Chile, Minsal. Estrategia nacional de salud para el cumplimiento de los objetivos sanitarios de la década 2011-2020. 2011. Accedido 31 de marzo de 2012. Available from: http://www.minsal.gob.cl/portal/ url/item/b89e911085a830ace0400101650115af.pdf.

16. White H, Glazier R. Do hospitalist physicians improve the quality of inpatient care delivery? A systematic review of process, efficiency and outcome measures. BMC Med 2011; 9: 58.

17. Rifkin W, Burger A, Holmboe E, Sturdevant B. Compa- rison of hospitalists and nonhospitalists regarding core measures of pneumonia care. Am J Manag Care 2007; 13 (3): 129-32.

18. Lindenauer P, Chehabeddine R, Pekow P, Fitzgerald J, Benjamin E. Quality of care for patients hospitalized with heart failure: assessing the impact of hospitalists. Arch Intern Med 2002; 162 (11): 1251-6.

19. Roytman M, Thomas S, Jiang C. Comparison of practice patterns of hospitalists and community physicians in the care of patients with congestive heart failure. J Hosp Med 2008; 3 (1): 35-41.

20. Somekh N, Rachko M, Husk G, Friedmann P, Bergmann S. Differences in diagnostic evaluation and clinical outcomes in the care of patients with chest pain based on admitting service: the benefits of a dedicated chest pain unit. J Nucl Cardiol 2008; 15 (2): 186-92.

21. Schneider J, Zhang Q, Auerbach A, Gonzales D, Kaboli $\mathrm{P}$, Schnipper J, et al. Do hospitalists or physicians with greater inpatient HIV experience improve HIV care in the era of highly active antiretroviral therapy? Results from a multicenter trial of academic hospitalists. Clin Infect Dis 2008; 46 (7): 1085-92.

22. Torres J. Estudio acerca del uso y la gestion de camas hospitalarias en la Región Metropolitana. Accedido 15 de marzo de 2012. Available from: http://www.saludyfuturo.cl/documentos/GESTIONCAMAS.pdf.

23. Dobrzanska L, Newell R. Readmissions: a primary care examination of reasons for readmission of older people and possible readmission risk factors. J Clin Nurs 2006; 15 (5): 599-606.

24. Boosting Care Transicions Room Resource. Risk Assessment Tool: the 8Ps. 2008. Accedido 15 de marzo de 2012. Available from: http://www.hospitalmedicine. org/ResourceRoomRedesign/RR_CareTransitions/ html_CC/06Boost/03_Assessment.cfm.

25. Rifkin W, Conner D, Silver A, Eichorn A. Comparison of processes and outcomes of pneumonia care between hospitalists and community-based primary care physicians. Mayo Clin Proc 2002; 77 (10): 1053-8.

26. Scheurer D, Miller J, Blair D, Pride P, Walker G, Cawley P. Hospitalists and improved cost savings in patients with bacterial pneumonia at a state level. South Med J 2005; 98 (6): 607-10.

27. Auerbach A, Wachter R, Katz P, Showstack J, Baron R, Goldman L. Implementation of a voluntary hospitalist service at a community teaching hospital: improved clinical efficiency and patient outcomes. Ann Intern Med 2002; 137 (11): 859-65.

28. Meltzer D, Manning W, Morrison J, Shah M, Jin L, Guth T, et al. Effects of physician experience on costs and outcomes on an academic general medicine service: 
results of a trial of hospitalists. Ann Intern Med 2002; 137 (11): 866-74.

29. Wachter R, Goldman L. The emerging role of "hospitalists" in the American health care system. N Engl J Med 1996; 335 (7): 514-7.

30. Hernández A, Greiner M, Fonarow G, Hammill B, Heidenreich P, Yancy C, et al. Relationship between early physician follow-up and 30-day readmission among Medicare beneficiaries hospitalized for heart failure. JAMA 2010; 303 (17): 1716-22.

31. Centers for Disease Control and Prevention. Monitoring hospital-acquired infections to promote patient safetyUnited States, 1990-1999. MMWR Morb Mortal Wkly Rep 2000; 49 (8): 149-53.

32. O'Grady N, Alexander M, Burns L, Dellinger E, Garland J, Heard S, et al. Guidelines for the prevention of intravascular catheter-related infections. Am J Infect Control 2011; 39 (4 Suppl 1): S1-34.

33. Rebmann T, Greene L. Preventing catheter-associated urinary tract infections: An executive summary of the Association for Professionals in Infection Control and Epidemiology, Inc, Elimination Guide. Am J Infect Control 2010; 38 (8): 644-6.

34. Rebmann T, Greene L, Preventing ventilator-associated pneumonia: An executive summary of the Association for Professionals in Infection Control and Epidemiology, Inc, Elimination Guide. Am J Infect Control 2010; 38 (8): 647-9.

35. Chung P, Morrison J, Jin L, Levinson W, Humphrey $\mathrm{H}$, Meltzer D. Resident satisfaction on an academic hospitalist service: time to teach. Am J Med 2002; 112 (7): 597-601.

36. Kulaga M, Charney P, O’Mahony S, Cleary J, McClung $\mathrm{T}$, Schildkamp $\mathrm{D}$, et al. The positive impact of initiation of hospitalist clinician educators. J Gen Intern Med 2004; 19 (4): 293-301.

37. Hauer K, Wachter R, McCulloch C, Woo G, Auerbach A. Effects of hospitalist attending physicians on trainee satisfaction with teaching and with internal medicine rotations. Arch Intern Med 2004; 164 (17): 1866-71.

38. Kripalani S, Pope A, Rask K, Hunt K, Dressler D, Branch
W, et al. Hospitalists as teachers. J Gen Intern Med 2004; 19 (1): 8-15.

39. Hunter A, Desai S, Harrison R, Chan B. Medical student evaluation of the quality of hospitalist and nonhospitalist teaching faculty on inpatient medicine rotations. Acad Med 2004; 79 (1): 78-82.

40. Natarajan P, Ranji S, Auerbach A, Hauer K. Effect of hospitalist attending physicians on trainee educational experiences: a systematic review. J Hosp Med 2009; 4 (8): 490-8.

41. Huddleston J, Long K, Naessens J, Vanness D, Larson $\mathrm{D}$, Trousdale $\mathrm{R}$, et al. Medical and surgical comanagement after elective hip and knee arthroplasty: a randomized, controlled trial. Ann Intern Med 2004; 141 (1): 28-38.

42. Phy M, Vanness D, Melton L, Long K, Schleck C, Larson $\mathrm{D}$, et al. Effects of a hospitalist model on elderly patients with hip fracture. Arch Intern Med 2005; 165 (7): 796801.

43. Roy A, Heckman M, Roy V. Associations between the hospitalist model of care and quality-of-care-related outcomes in patients undergoing hip fracture surgery. Mayo Clin Proc 2006; 81 (1): 28-31.

44. Wachter R, Katz P, Showstack J, Bindman AB, Goldman L. Reorganizing an academic medical service: impact on cost, quality, patient satisfaction, and education. JAMA 1998; 279 (19): 1560-5.

45. Peterson M. A systematic review of outcomes and quality measures in adult patients cared for by hospitalists vs nonhospitalists. Mayo Clin Proc 2009; 84 (3): 248-54.

46. Gregory D, Baigelman W, Wilson I. Hospital economics of the hospitalist. Health Serv Res 2003; 38 (3): 905-18; discussion 919-22.

47. Schroeder S, Schapiro R. The hospitalist: new boon for internal medicine or retreat from primary care? Ann Intern Med 1999; 130 (4 Pt 2): 382-7.

48. Sharma G, Freeman J, Zhang D, Goodwin J. Continuity of care and intensive care unit use at the end of life. Arch Intern Med 2009; 169 (1): 81-6.

49. Wachter R. An introduction to the hospitalist model. Ann Intern Med 1999; 130 (4 Pt 2): 338-42. 